\title{
Pallet Sorting using Optical Character Recognition
}

\author{
Suraj Muley ${ }^{1}$, Virendra Patil ${ }^{2}$, Payal Dhage ${ }^{3}$, Sarika Panwar $^{4}$ \\ E\&TC Department, Smt. Kashibai Navale College of Engineering ${ }^{1,2,3,4}$
}

\begin{abstract}
Although, automation led us into the development in terms of sophistication of human efforts in bringing out mass production of more precisely manufactured goods but it needs to be modified to be a smarter intelligent system. Pallet sorting equipment using OCR techniques reduces human efforts in recognizing required position of object. It reduces time required to sort objects with less time. Different preprocessing techniques are used like skew correction, binarization, text recognition etc. In this paper, the proposed system is based on an efficient binarization and enhancement technique followed by a suitable connected hardware assembly is discussed. The objective of the study is to significantly improve the capabilities of pallet sorting by address recognition.
\end{abstract}

Keywords: OCR, skew correction, text recognition, pallet sort.

\section{INTRODUCTION}

In almost all industry sorting is essential which reduces human efforts and sort the object according to its required position within less time. Images captured by the camera contains the text information which is often required to be automatically recognized and processed. The outputs of the system are completely reliable which can be further linked with huge working systems. This paper directs toward methodology which deals with automatic detection, segmentation and recognition of visual text entities in complex images. It may be any textual part of the scene images such as name plates or even text appearing on T-shirts or pallets. The research field of text recognition receives a growing attention due to the evolution of digital cameras and the great variety of potential applications. Such applications include robotic vision, image retrieval, intelligent navigation systems and the visual challenged persons.

Images usually suffer from low resolution and low quality, perspective distortion and complex background. Image text is hard to detect, extract and recognize since it can appear with any slant, tilt, in any lighting, upon any surface and may be partially concealed. Many approaches for detection of text from natural scene images have been proposed recently.

\section{LITERATURE SURVEY}

The technical paper by Suny b. et al[1] includes technical report of the Advanced Character Recognition Project conducted at the State University of New York. Experimental Investigation suggest that, Current commercial OCR techniques involve three successive steps. These are image processing, character classification and contextual postprocessing. Scanning Hardware uses Laser and semiconductor array scanners, which were reviewed for application to postal information processing. These technologies were chosen for study because of their ability to provide the required resolution and throughput specifications. During the first run, the machine reported52\% correct performance, during the second, $92 \%$ correct performance. This reflects the differences because the first run contained handwritten, typed, and foundry font addresses and the second had well-prepared mail from a few sources. Reasons for rejection are summarized below: skewed address, touching characters, portion of address obscured in a window envelope smeared characters caused by ink-jet or dot matrix printing, hand-generated address

The paper published by AyatullahFarukMollah et al [2], presents a complete Optical Character Recognition (OCR) system for camera captured image/graphics embedded textual documents for handheld devices. At first, text regions are extracted and skew corrected. Then, these regions are binarized and segmented into lines and characters. Characters are passed into the recognition module. Experiments have been carried out to evaluate the performance and applicability of the current technique for character. The images have been captured with a cell phone camera. In the present experiment only a few text regions are improperly segmented. It may be noted that the present segmentation technique is not meant for italic and cursive texts. So, such texts have been ignored .It means that no instance is found where two or more text lines have been extracted as a single text line or a single text line gets treated as more than one text lines.

Another paper by from International Journal of Advances in Electronics and Computer Science [3] discusses automation for sorting using raspberry pi3 in which it is shown that colour objects are getting sorted according to its conditions. Author suggest that the system used is cost effective, less time consuming and technically simple approach to sort the objects. But further approaches can be done such that to increase the capability of sorting system. Also the 
proposed system can be used only when the sorting having colours. It cannot detect the textual context written on the sortable objects.

\section{METHODOLOGY}

The proposed system works on the principle of OCR using raspberry pi processor using camera module of raspberry pi. The block diagram for the proposed system given as follows:

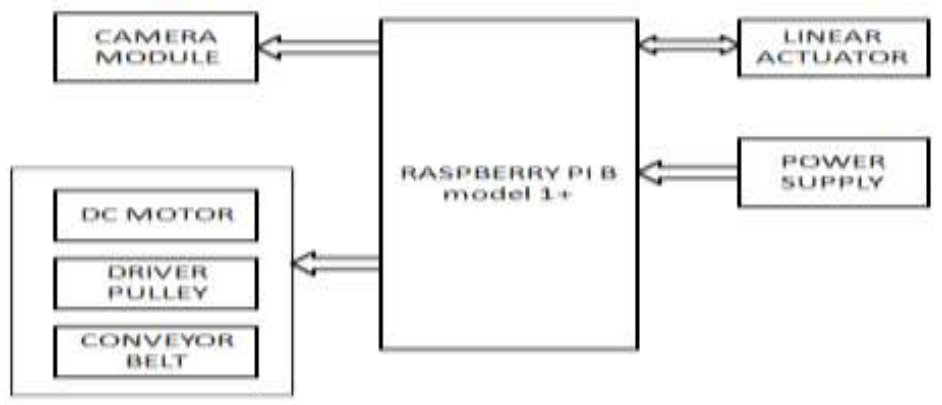

Figure: 1. Block diagram of system.

After switching on the mechanical assembly consisting of conveyor belt and pallet the object starts moving towards the camera module. The image captured by the camera preprocesses that image. Operations like filtering, skew correction and text recognition to convert the image into the textual format.

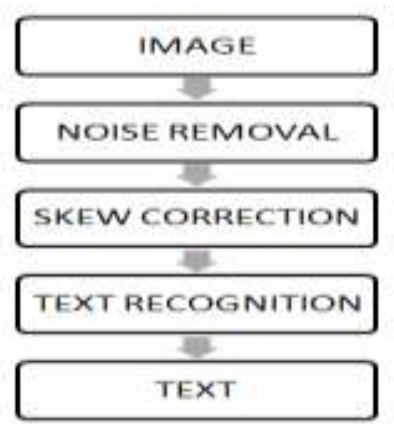

Figure: 2. Pre-Processing steps of OCR

Noise removal block does filtering using median filter. This filter is used because the median filter is effective for removing impulse noise such as "salt and pepper noise" which is random occurrences of black and white pixels. The next process is of correcting the skew present in the image the method we employed was to project the page at several angles, and determine the variance in the number of black pixels per projected lines. Somewhat less accurate, but faster and works pretty well with slight skews that are common in scanned images. Text recognition extracts the desired text from the actual scanned image and recognize characters present in the image.

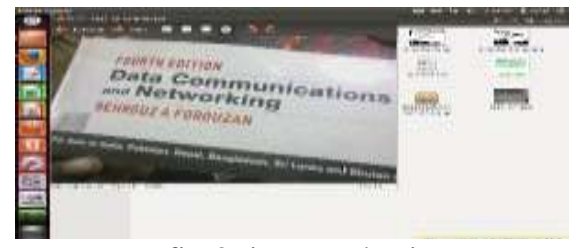

fig.3: input color image

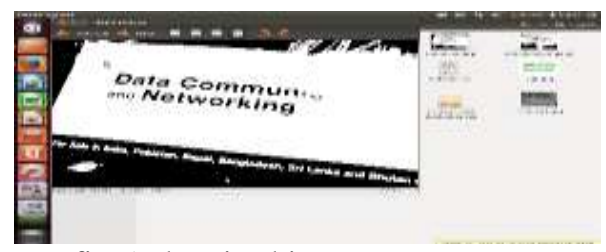

fig.5: denoised image

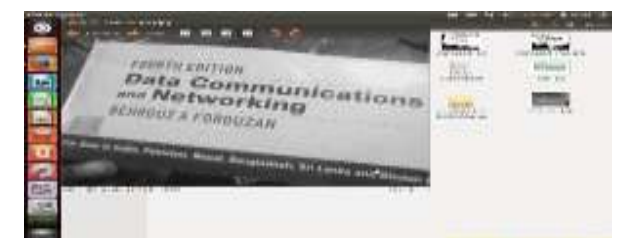

fig.4: gray image

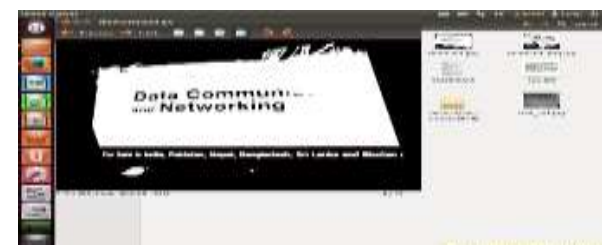

fig.6: skew corrected image 


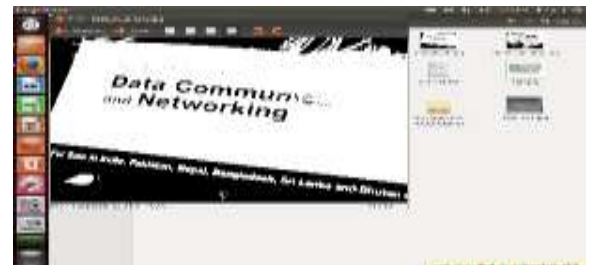

fig.7: binary image

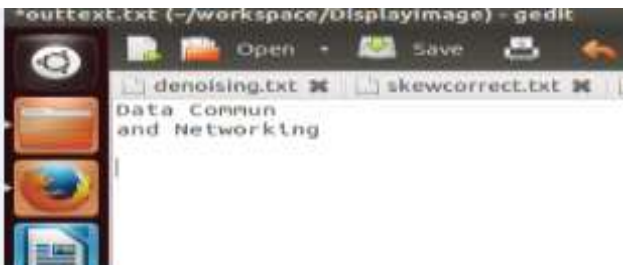

fig.8: text output

After preprocessing and scanning of object the characters of the textual output will be compared with the database of the processor. According to the database if the text is present it will get sorted by the servo motor used at the end of the mechanical assembly and drop it to the desired location of that object.

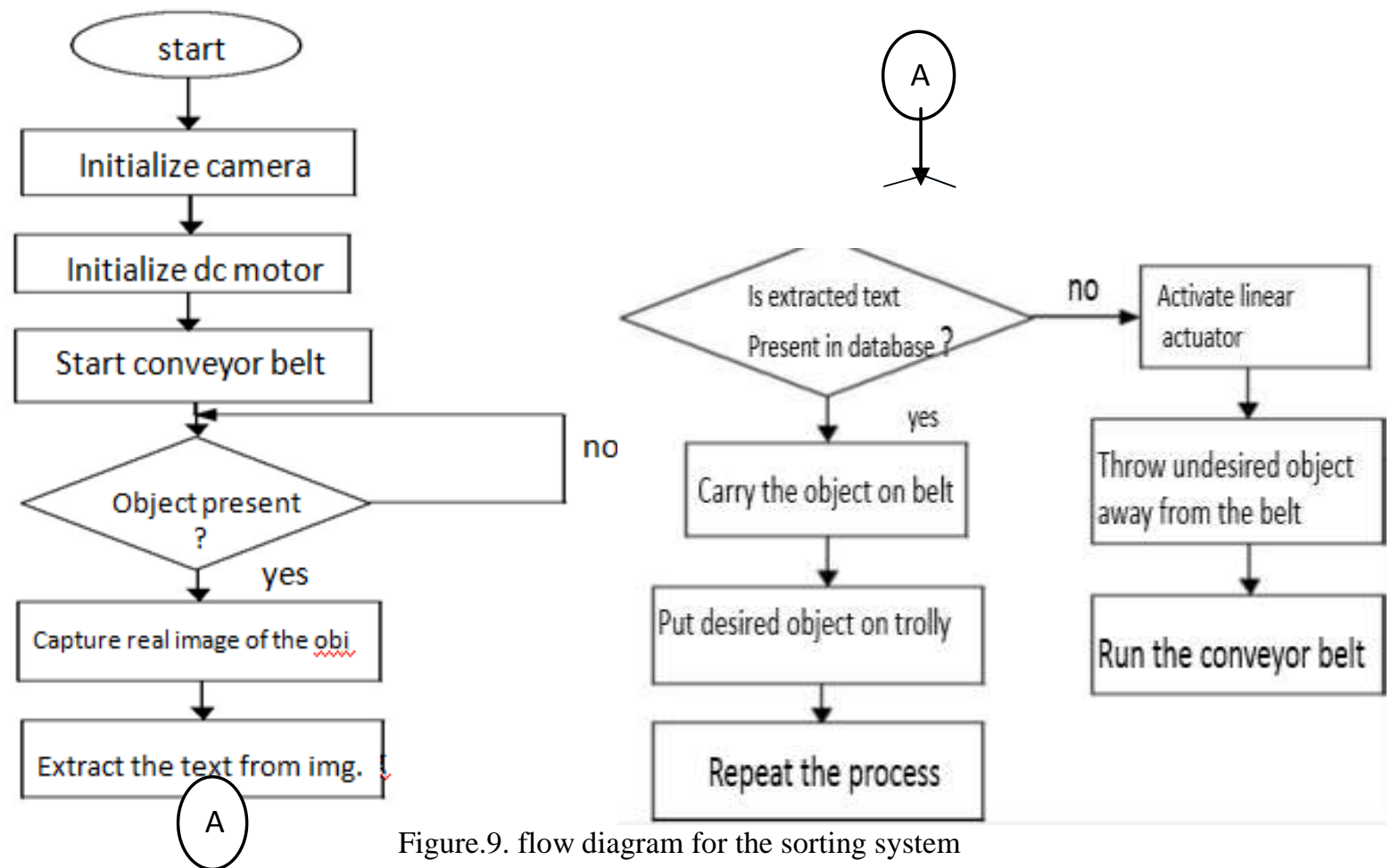

The hardware of the proposed system for implementation:

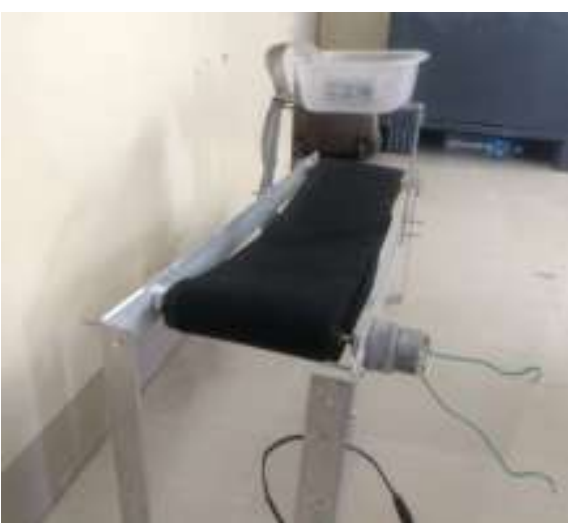

Figure: 10. Conveyor belt assembly

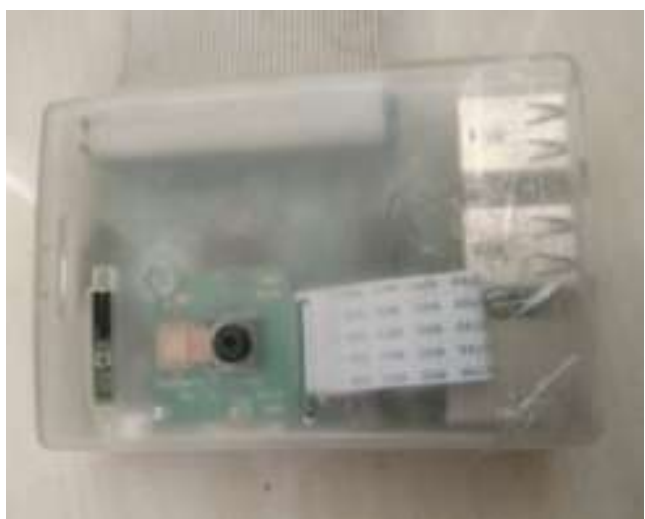

Figure: 11. Raspberry pi \& camera module

\section{CONCLUSION}

In this paper, Pallet sorting is done using Optical Character Recognition. It is observed that this method is more efficient than pattern recognition or color recognition process. 


\section{IJARCCE}

Because of correct \& efficient text recognition, this technique can be used in many industrial applications \& can be modified as per the requirement of user.

\section{REFERENCES}

[1] Optical Character Recognition Techniques: A Survey, Journal of Emerging Trends in Computing and Information Sciences, Vol. 4, No. 6 June 2013, Sukhpreet Singh

[2] Optical Character Recognition Techniques: A Review, International Journal of Advanced Research in Computer Science and Software Engineering, Volume 4, Issue 5, May 2014,Er. Neetu Bhatia

[3] Forty years of research in character and document recognition---an industrial Perspective, Hiromichi Fujisawa, Central Research Laboratory, Hitachi, Ltd., 1-280 Higashi-koigakubo, Kokubunji, Tokyo 185-8601, Japan.

[4] An overview of the Tesseract OCR engine, Ray SmithGoogle Inc.

[5] Automation for sorting of objects using raspberryPi 3 1sheela.s, 2meghashree. S, 3monica. L, 4prathima. A, 5shriya. M. Kumar 1asst. Professor, department of tce, dbit. 2,3,4,5ug scholar, department of tce, dbit, bangalore.

[6] Automated Object Sorting Using Raspberry Pi N.Aarthi1, P.Sahithi2, P.V.Sitaramaih3, M.Indu Vardhani4, N. Ranjith Kumar5, D. Suneel Varma6 1,2,3,4,5,6(Electronics and Communication Engineering, Bapatla Engineering College(Autonomous), AcharayaNagarjuna University, India. 